\title{
Migraine patients exhibit abnormalities in the visual evoked potential
}

\author{
J F CONNOLLY, ${ }^{*}$ M GAWEL,$\dagger$ F CLIFFORD ROSE $\ddagger$ \\ From the Department of Psychiatry, ${ }^{*}$ and the Princess Margaret Migraine Clinic, $†$ Department of Neurology, \\ Charing Cross Hospital, London
}

SUMMARY An experiment is described which compared visual evoked potential (VEP) amplitudes and latencies in normal subjects and migraine patients. Several VEP abnormalities were found: at vertex and to a lesser extent at temporal sites, P100-N120 amplitudes were larger in patients; at vertex and temporal sites N120 amplitudes were larger in patients; at temporal sites patents had larger N120-P200 amplitudes but smaller P100 amplitudes. Peak latencies of the VEP were also found to be abnormal in patients. At vertex, patients had delayed N120 peak latencies while at temporal sites delays were found in the P200 latencies. Patients were subdivided according to side of headache. Right-sided headache patients showed larger temporal P100 amplitudes and larger left temporal P100-N120 amplitudes than bilateral headache patients.

Visual evoked potentials (VEP) have been used in clinical neurology for the diagnosis of lesions in the optic pathway, and more specifically in the diagnosis of multiple sclerosis. ${ }^{1}$ Although alterations in evoked potentials can be due to marked anatomical changes, for example, ischaemic damage to the nervous system, ${ }^{4}$ other factors include neurotransmitter abnormalities, for example as in Parkinsonism $^{2}$ and phenylketonuria, ${ }^{3}$ when the changes tend to correlate with the severity of the clinical condition. More subtle abnormalities have been reported in conditions not usually associated with signs of overt neurological damage such as migraine (homonymous hemianopia pathology) and following the ingestion of a number of psychoactive drugs. ${ }^{5}$

It has been proposed that there is a failure of input modulation in migraine: Wolff $s^{6}$ early experiment suggested that migraineurs had a low pain threshold and Sicuteri ${ }^{7}$ postulated a central deficiency of a sensation modulatory substance. His experiments concentrated initially on

Address for reprint requests: Dr F Clifford Rose, Princess Margaret Migraine Clinic, Department of Neurology, Charing Cross Hospital, Fulham Palace Rd, London W6 8RF, UK.

Received 28 August 1981 and in revised form 16 November 1981. Accepted 17 January 1981 5-hydroxytryptamine (5HT) metabolism and he showed that hallucinogenic responses of migraineurs to lysergic acid diethylamide (LSD), a 5HT antagonist, were present at much lower dose levels than those of controls. More recently, Sicuteri ${ }^{8}$ has proposed that substance $P$ as well as 5HT is important in maintaining the "anti nociceptive system".

The symptomatology of migraine is mediated by vascular events, the neurological signs and symptoms probably being related to focal ischaemia, while the pain is related to extra cerebral vasodilatation. The studies of cerebal blood flow by Sakai and Meyer' ${ }^{9}$ suggest that there are areas of focal ischaemia surrounded by hyperaemia in the brain during a migraine attack, and Welch's ${ }^{10}$ finding of elevation of gamma amino butyric acid (GABA) and cyclic AMP following an attack suggests that cerebral ischaemia does occur. The ischaemic processes during the attack have been studied by MacLean and Appenzeller (1975) ${ }^{11}$ and by Regan and Heron (1970), ${ }^{12}$ both of whom found alteration of the VEP amplitude over the hemisphere presumed to be suffering from ischaemia. Kennard et al studied visual evoked potentials in migraine patients between attacks using a checkerboard stimulus and found that the latency of the major positive wave was greater and the amplitude larger than in a group 
of age-matched controls; they interpreted their results as being indicative of a neurotransmitter abnormality resulting in either a lack of inhibition or an increase in excitation. We undertook the present study in order to evaluate this phenomenon further and to assess the nature of the VEP abnormalities in migraine patients.

\section{Methods}

The control group consisted of 22 healthy volunteers (11 men and 11 women) aged from 21 to 48 years. The patient group consisted of 16 migraineurs (both classical and common, some of whom experienced visual symptoms) (15 women and one man), ranging in age from 16 to 61 years. All patients and controls were dextral and had normal, or corrected normal, vision. The history of migraine ranged from 2 to 15 years with an average frequency of attacks of one per month. None of the patients had experienced an attack during the week prior to testing. The patient groups were subdivided according to the side of the headache, and this resulted in four left-sided headache, six right-sided headache, and six subjects with bilaterial headache. For technical reasons, VEP data recorded from temporal lobe sites was available for only 12 of the 16 patients and there were six right-sided headache and six bilateral headache subjects. Patients had no treatment prior to testing.

The stimuli consisted of unpatterned flashes at six intensities: $0.09,0.18,0.35,0.70,1.40,2.80 \mathrm{~cd} / \mathrm{m}^{2}(0.31,0.65$, $1 \cdot 25,2 \cdot 5,5 \cdot 0$ and $10 \cdot 0 \mathrm{ft}$ lamberts); the stimuli subtended an angle of $20^{\circ}$ horizontal and $16^{\circ}$ vertical, and were presented in blocks of ten for each intensity to a total of 360 presentations. The blocks were presented according to a Williams Latin square design. ${ }^{13}$ Stimulus duration was 500 $\mathrm{ms}$ and the interstimulus interval (ISI) was $500 \mathrm{~ms}$. The interval between the blocks of stimuli was variable between 5 and $15 \mathrm{~s}$. EEG was recorded on a Grass polygraph with the half amplitude high frequency filter at $75 \mathrm{~Hz}$ and a time constant of $0 \cdot 1 \mathrm{~s}$. Subjects were tested with eyes open and fixated on a point in the middle of the stimulus field. EEG was recorded, with $\mathrm{Ag} / \mathrm{AgCl}$ electrodes fixed to the scalp with collodion, from $\mathrm{Cz}, \mathrm{T} 3$, and $\mathrm{T} 4$ referred to linked ears, and were grounded with an electrode placed on the right arm. Electrode impedances were equalised and kept below $5 \mathrm{~K} \Omega$. Vertical and horizontal eye movements were monitored by electro-oculography (EOG). ${ }^{14}$ In no subject were more than $10 \%$ of trials lost due to EOG artefacts, and no relation was found between EOG artefact and stimulus intensity. Each EEG channel was sampled at $250 \mathrm{~Hz}$ for $20 \mathrm{~ms}$ prestimulus and $500 \mathrm{~ms}$ poststimulus and stored directly on a PDP 11/04. Evoked potentials were checked visually and then scored automatically by computer. Manual scoring using a cursor programme was used in those cases where the triphasic waveform (that is, $\mathrm{P} 100$, N120, P200) of the VEP was too small or ill-defined for automatic scoring. In such cases $\mathrm{N} 120$ was defined as the most negative peak in the latency range of 80 to $200 \mathrm{~ms}$ while P100 was the most positive peak between N120 and 280 ms. P100-N120 and N120-P200 peak-to-trough amplitudes were measured, as well as the amplitude of each (that is, P100, N120 and P200) from the mean prestimulus baseline level. Latencies of each peak were also calculated.

\section{Results}

A summary of the VEP amplitude results statistically assessed with analysis of variance is presented in the table. It should be noted that no sex effects were found in the control group. Migraineurs showed a larger P100-N120 peak-to-trough amplitude. Further analysis showed this effect was due primarily to the N120 peak, which was substantially larger in the migraine group. The two groups did not differ on any other amplitude measures. Analysis of the latency data revealed that it was again the N120 peak which proved to be abnormal $(p<0.05)$. The migraine group exhibited delayed latencies, particularly to the brighter flashes of light (fig (a)). When recorded from vertex neither the P100 nor the P200 peaked latencies were abnormal in the migraine group. The migraine group also showed abnormalities in the VEP when recorded from temporal placements. There was a trend for migraineurs to have larger P100-N120 amplitudes, and they showed significantly larger N120-P200 amplitudes compared to normal controls. Results for the individual peaks revealed that migraineurs showed smaller P100 amplitudes but larger N120 amplitudes. Results for peak latencies showed that at temporal sites it was the P200 peak which was delayed in the migraine example $(p<0.0264)$. Observation of the raw data for three of the major amplitude results (fig (b)) shows that only one or at the most two normal subjects exhibit amplitude larger than the mean of the migraine group. When the migraine group was analysed according to side of headache, only the temporal sites revealed abnormality in the VEP. Those patients who experienced right-sided headaches showed larger P100-N120 amplitudes (p $<0.0491)$ at the left temporal site compared to the bilateral headache group. Also, the right-sided headache group showed larger temporal P100 amplitudes (that is, $T_{3}$ and $T_{4}$ amplitudes averaged together) compared to the bilateral headache group $(p<0.404)$.

\section{Discussion}

The results indicate that on some measures of the visual evoked response the migraine patients differ from controls, specifically the larger amplitudes of response of the N120 wave both as measured from electrical baseline and as P100-N120 peak-totrough amplitude. This is a similar finding to that reported by Kennard et $a^{5}$ who used checkerboard stimulation and found that the P120 amplitude was 


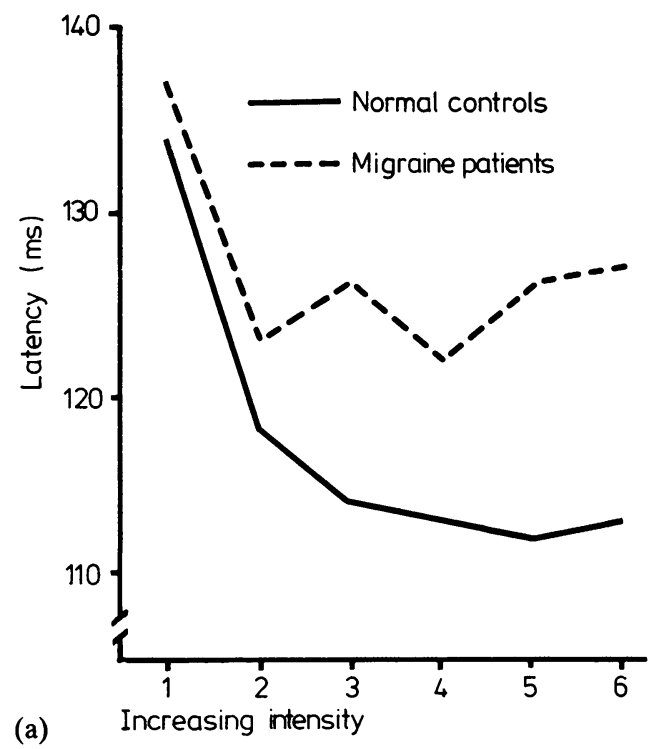

Figure (a) Latency of the vertex N120 peak across the six stimulus intensities. (b) Plot of individual amplitudes for subjects in the migraine and normal control groups. From left: vertex P100-N120 amplitudes; vertex N120 amplitudes; temporal N120 amplitudes.

(a) Increasing intensity 
Table Peak and peak-to-trough amplitudes (in $\mu V$ ) for normal controls and migraine patients for the visual event-related potential recorded from vertex $(C z)$ and temporal $\left(T_{3}\right.$ and $\left.T_{4}\right)$ sites. The standard error of the mean appears in parentheses and the statistical significance is presented in the centre column

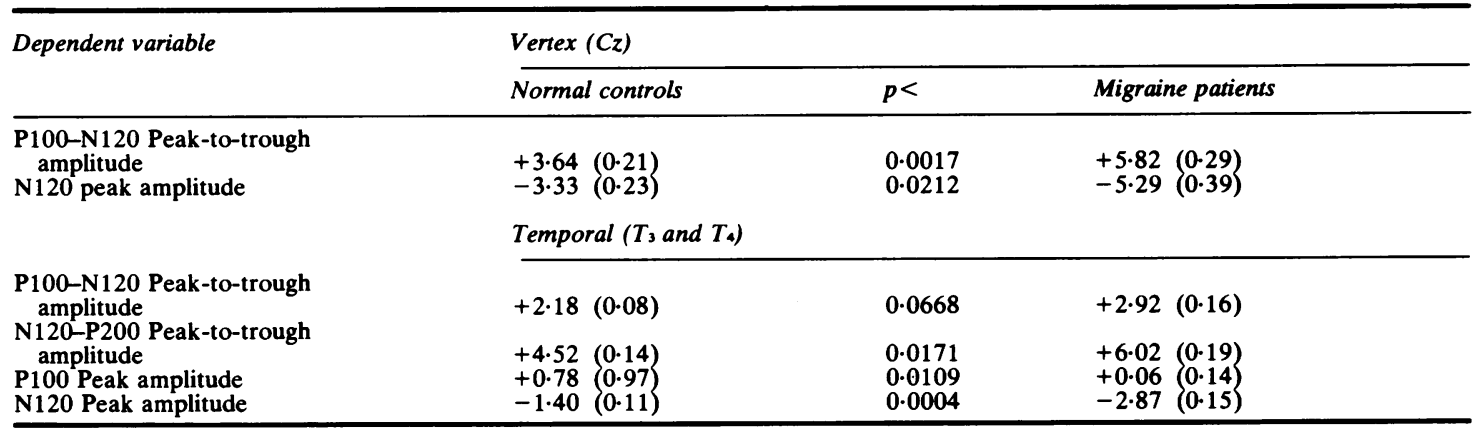

greater in the migraine patients, both taken as the N1(N80) P2(P100) amplitude, and as the P100N120 amplitude. Kennard $e t$ al $^{\text {s }}$ also found a prolonged P100 latency; we were unable to confirm this, but did find a prolonged $\mathrm{Cz}: \mathrm{N} 120$ latency to the brighter flashes of light as well as a prolonged P200 latency at temporal sites. Clinically many patients are intolerant of noises and bright lights and some find these can even precipitate an attack. These results may suggest a neurophysiological basis for a different responsiveness of the visual system in migraine patients. The asymmetrical response of the P100-N120 amplitude in the patients with different sides of headache is interesting. It may reflect a secondary phenomenon to repeated right-sided headaches affecting structures in the left hemisphere. However, further work is needed particularly with a left-sided headache patient sample before any interpretation can be usefully proffered.

JFC is the holder of a Wellcome Research Fellowship.

\section{References}

${ }^{1}$ Halliday AM, McDonald WI, Mushin J. The visual evoked response in the diagnosis of multiple sclerosis. Br Med J 1973;iv:661-4.

${ }^{2}$ Gawel M, Das P, Vincent S, Rose FC. Visual and auditory evoked potentials in Parkinson's disease. J Neurol Neurosurg Psychiatry 1981;44:227-32.

${ }^{3}$ Schafer EW, McKean CM. Evidence that monoamines influence human evoked potentials. Brain Res 1975:99:49-58.

4 Cohn R. Evoked visual cortical responses in homony- mous hemianopia defect in man. Electroencephalogr Clin Neurophysiol 1963;15:922.

' Kennard C, Gawel M, Rudolph N de M, Rose FC. Visual evoked potentials in migraine subjects. In: Friedman AP, Granger M, Critchley M, eds. Research and Clinical Studies in Headache 6:72-80. Basel: Karger, 1978.

'Dalessio DJ. Wolffs Headache and Other Head Pains. New York: Oxford University Press, 1980;89.

${ }^{7}$ Sicuteri F. Headache as a possible expression of a deficiency of brain 5-hydroxytryptamine. Headache 1972;12:69-72.

- Sicuteri F, Anselmi B, Del Brasseo PL. Systemic non organic central pain. A new syndrome with decentralisation supersensitivity. Headache 1978;18:133-6.

- Sakai F, Stirling Meyer J. Regional cerebral haemodynamine during migraine and cluster headache measured by the Xe 133 inhalation method. Headache 1978;18:122-32.

${ }^{10}$ Welch KMA, Chabi E, Nell J, Bartosh K, Meyer JS, Matthew NT. Similarities in biochemical effects of cerebral ischaemic patients with cerebrovascular disease and migraine. In: Raymond Green, ed. Current Concepts on Migraine Research. New York: Raven Press, 1978:1-9.

${ }^{11}$ MacLean C, Appenzeller O, Cordourdo JT, Rhodes J. Flash evoked potentials in migraine. Headache 1975;14:193-8.

12 Regan D, Heron JR. Simultaneous recording of visual evoked and neutrals from the left and right hemisphere in migraine. In: Cochrane T, ed. Background to Migraine. London Heinemann, 1970;66.

${ }^{13}$ Edwards AL. Experimental Design in Psychological Research (3rd ed.) New York: Holt, Rinehard and Winston, 1968.

${ }^{14}$ Connolly JF, Kleinman KH. A single channel method for recording vertical and lateral eye movements. Electroencephalogr Clin Neurophysiol 1978;45:1289. 